\title{
Influence of the contact-impact force model on the dynamic response of multi-body systems
}

\author{
P Flores $^{1 *}$, J Ambrósio ${ }^{2}$, J C P Claro ${ }^{1}$, and H M Lankarani ${ }^{3}$ \\ ${ }^{1}$ Departamento de Engenharia Mecânica, Universidade do Minho, Guimarães, Portugal \\ ${ }^{2}$ Instituto de Engenharia Mecânica (IDMEC), Instituto Superior Técnico, Lisboa, Portugal \\ ${ }^{3}$ Mechanical Engineering Department, Wichita State University, Kansas, USA
}

The manuscript was received on 7 January 2005 and was accepted after revision for publication on 6 October 2005.

DOI: 10.1243/146441906X77722

\begin{abstract}
This work deals with contact-impact force models for both spherical and cylindrical contact surfaces. The incorporation of the friction phenomenon, based on the Coulomb friction law, is also discussed together with an effective computational strategy, which includes the automatic step size selection procedure. Impacts within a revolute clearance joint in a basic slider-crank mechanism are used as an example to compare the different contact force models. The collision is a prominent phenomenon in many multi-body systems such as mechanisms with intermittent motion, kinematic discontinuities, and clearance joints. As a result of an impact, the values of the system state variables change very fast, eventually looking like discontinuities in the system velocities and accelerations. The impact is characterized by large forces that are applied and removed in a short time period. The knowledge of the peak forces developed in the impact process is very important for the dynamic analysis of multibody systems and it has consequences in the design process. The model for the contactimpact force must consider the material and geometric properties of the colliding surfaces, consider information on the impact velocity, contribute to an efficient integration, and account for some level of energy dissipation. These characteristics are ensured with a continuous contact force model, in which the deformation and contact forces are considered as continuous functions.
\end{abstract}

Keywords: contact-impact forces, friction forces, multi-body dynamics

\section{INTRODUCTION}

Impact occurs in the collision of two or more bodies, which can be unconstrained or may belong to a multi-body system. The impact phenomenon is characterized by abrupt changes in the values of system variables, most visible discontinuities in the system velocities. Other effects directly related to the impact phenomena are the vibration propagation on the system components, local elastic/plastic deformations at the contact zone, and energy dissipation. The impact is a very important phenomenon in many mechanical systems such as

* Corresponding author: Departamento de Engenharia Mecânica, Universidade do Minho, Campus de Azurém, 4800-058 Guimarães, Portugal. mechanisms with intermittent motion and clearance joints $[\mathbf{1}, 2]$. The selection of the most adequate contact force model plays a key role in the correct design and analysis of these kinds of mechanical systems.

By and large, an impact may be considered to occur in two phases: the compression or loading phase and the restitution or unloading phase. During the compression phase, the bodies deform in the normal direction to the impact surface and the relative velocity of the contact points/surfaces on the bodies in that direction is gradually reduced to zero. The end of the compression phase is referred to as the instant of maximum compression or maximum penetration. The restitution phase starts at this point and ends when the bodies separate from each other $[3]$. The restitution coefficient reflects the type of collision. For a fully elastic contact, the 
restitution coefficient is equal to the unit, whereas for a fully plastic contact, the restitution coefficient is null. The most general and predominant type of collision is the oblique eccentric collision, which involves both relative normal velocity and relative tangential velocity $[\mathbf{4}, \mathbf{5}]$.

In order to evaluate efficiently the contact-impact forces resulting from collisions in multi-body systems, such as the contact between the bearing and journal in a revolute joint with clearance, special attention must be given to the numerical description of the contact force model. Information on the impact velocity, material properties of the colliding bodies, and geometry characteristics of the contact surfaces must be included into the contact force model. These characteristics are observed with a continuous contact force, in which the deformation and contact forces are considered as continuous functions [6]. Furthermore, it is important that the contact force model can add to the stable integration of the multi-body system's equation of motion.

In a broad sense, there are two different methods to solve the impact problem in multi-body systems, namely, the continuous and discontinuous approaches [6]. Within the continuous approach, the methods commonly used are the continuous force model, which is in fact a penalty method, and the unilateral constraint methodology, based on the linear complementary approach [7]. The continuous contact force model represents the forces arising from collisions and assumes that the forces and deformations vary in a continuous manner. In this method, when contact between the bodies is detected, a force perpendicular to the plane of collision is applied. This force is typically applied as a spring-damper element, which can be linear, such as the Kelvin-Voigt model [8], or non-linear, such as the Hunt and Crossley model [9]. For long impact durations, this method is effective and accurate in that the instantaneous contact forces are introduced into the system's equations of motion. The second continuous approach specifies that when contact is detected, a kinematic constraint is introduced into the system's equations. Such a constraint is maintained when the reaction forces are compressive and removed when the impacting bodies rebound from contact [10].

A second approach of a different nature, based on the Newton or Poisson hypotheses, is a discontinuous method that assumes that the impact occurs instantaneously being the integration of the equations of motion halted at the time of impact. Then, a momentum balance is performed to calculate the post-impact velocity. The integration is then resumed with the updated velocities until the next impact occurs. In the discontinuous method, the dynamic analysis of the system is divided into two intervals, before and after impact. The restitution coefficient is employed to quantify the dissipation of energy during the impact. The restitution coefficient only relates relative velocities after separation to relative velocities before contact and ignores what happens in-between. The discontinuous method is relatively efficient; however, the unknown duration of the impact limits its application, as for large enough contact periods, the system's configuration changes significantly [8]. Hence, the assumption of instantaneity of impact duration is no longer valid and the discontinuous analysis must not be adopted. This method, commonly referred to as piecewise analysis, has been used for solving the intermittent motion problem [1].

In dynamic analysis of multi-body systems with collisions, the deformation is known at every time step from the configuration of the system and the forces are evaluated based on the state variables. With the variation of the contact force during the contact period, the dynamic system's response is obtained by simply including updated forces into the equations of motion. As the equations of motion are integrated over the period of contact, this approach results in a rather accurate response. Furthermore, this methodology accounts for the changes in the system's configuration during the contact periods. In general, the contact points change during the collision. When there is no penetration between the colliding bodies, there is no contact and, consequently, the contact forces are null. The occurrence of penetration is used as the basis to develop the procedure to evaluate the local deformation of the bodies in contact. Although the bodies are assumed to be rigid, the contact forces correspond to those evaluated as if the penetration was due to local elastic deformations. These forces are calculated as being equivalent to those that would appear if the bodies in contact were pressed against each other by an external static force. This means that the contact forces are treated as elastic forces expressed as functions of the coordinates and velocities of the colliding bodies. The methodology used here allows for the accurate calculation of the contact points location. The direction of the normal force is determined from the normal vector to the plane of collision at the points of contact.

The impact within a revolute clearance joint in a basic slider-crank mechanism is used as an example to compare the different contact models. For effective computation, contact force model as well as the friction force law must be used together with an automatic time-step size selection procedure, such as the numerical predictor-corrector algorithm presented by Shampine and Gordon [11]. 


\section{NORMAL FORCE MODELS FOR SPHERICAL CONTACT SURFACES}

The simplest contact force relationship, known as Kelvin-Voigt viscous-elastic model, is modelled by a parallel spring-damper element [5]. The spring represents the elasticity of the contacting bodies and the damper describes the loss of kinetic energy during the impact. In general, the stiffness and damping coefficients have been assumed to be known parameters and the analysis have been confined to unconstrained bodies. The spring stiffness in the element can be calculated using a simple mechanical formula or obtained by means of the finite element method. Recently, Zhu et al. [12] proposed a theoretical formula for calculating damping in the impact of two bodies in a multi-body system. This model assumes that both the spring and damper are linear. When the contact bodies are separating from each other, the energy loss is included in the contact model by multiplying the rebound force with a coefficient of restitution. Thus, the restitution coefficient accounts for the energy dissipated during the impact in the form of a hysteresis in force-penetration relation.

The normal Kelvin-Voigt contact force, $F_{\mathrm{N}}$, is calculated for a given penetration depth, $\delta$, as

$$
F_{\mathrm{N}}=\left\{\begin{array}{ccl}
K \delta & \text { if } v_{\mathrm{N}}>0 & \text { (loading phase) } \\
K \delta c_{\mathrm{e}} & \text { if } v_{\mathrm{N}}<0 & \text { (unloading phase) }
\end{array}\right.
$$

where $K$ is the spring stiffness, $\delta$ the relative penetration depth, $c_{\mathrm{e}}$ the restitution coefficient, and $v_{\mathrm{N}}$ the relative normal velocity of the colliding bodies.

The primary drawback associated with this model deals with the quantification of the spring constant, which depends on the geometry and the physical properties of the contacting bodies. In contrast, the assumption of a linear relation between the penetration depth and the contact forces is at best a rough approximation because the contact force depends on the shape, conditions, and material properties of the contacting surfaces, all of which suggests a more complex relation.

For the linear Kelvin-Voigt model, Fig. 1 shows the penetration depth, $\delta$, the normal contact force, $F_{\mathrm{N}}$, and the hysteresis of two internally colliding spheres. The restitution coefficient and the spring stiffness are 0.9 and $1.5 \times 10^{8} \mathrm{~N} / \mathrm{m}$, respectively. The discontinuity that occurs in the impact force at maximum penetration is due to the decouple interpretation of the impact phenomenon using Poisson's interpretation, as illustrated in Fig. 1(b).

The best-known contact force law between two spheres of isotropic materials is due to the result of pioneering work by Hertz, which is based on the theory of elasticity [13]. The Hertz contact theory is restricted to frictionless surfaces and perfectly elastic solids. The Hertz law relates the contact force as a non-linear power function of penetration depth and can be expressed as [14]

$$
F_{\mathrm{N}}=K \delta^{n}
$$

where $K$ is a constant of proportionality and $\delta$ is the relative normal penetration between the spheres. The exponent $n$ is equal to 1.5 for circular and elliptical contacts. The parameter $K$ is dependent on the material properties and the shape of the contact, surfaces. For two spheres in contact the generalized stiffness coefficient is the function of the radii of the spheres $R_{i}$ and $R_{j}$ and the material properties as [15]

$$
K=\frac{4}{3\left(\sigma_{i}+\sigma_{j}\right)}\left[\frac{R_{i} R_{j}}{R_{i}+R_{j}}\right]^{1 / 2}
$$

where the material parameters $\sigma_{i}$ and $\sigma_{j}$ are given by

$$
\sigma_{k}=\frac{1-v_{k}^{2}}{E_{k}}, \quad k=i, j
$$

the quantities $v_{k}$ and $E_{k}$ are the Poisson ratio and the Young modulus associated with each sphere,

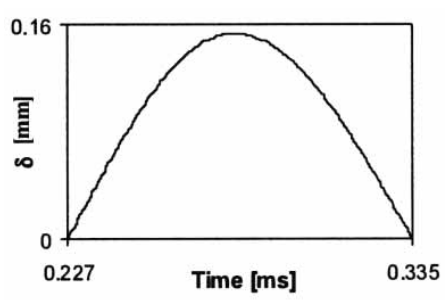

(a)

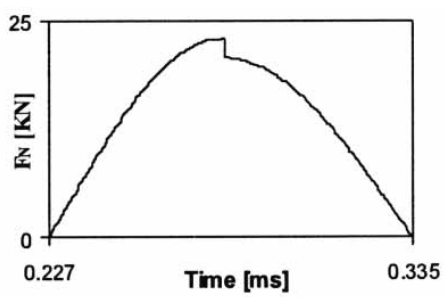

(b)

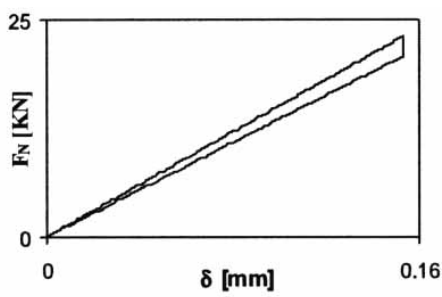

(c)

Fig. 1 Internally colliding spheres modelled by the linear Kelvin-Voigt viscous-elastic contact model: (a) penetration depth, $\delta$; (b) normal contact force, $F_{\mathrm{N}}$; (c) force-penetration relation 
respectively. For contact between a sphere body $i$ and a plane surface body $j$, the generalized stiffness coefficient depends on the radius of the sphere and the materials properties, being expressed by [15]

$$
K=\frac{4}{3\left(\sigma_{i}+\sigma_{j}\right)} \sqrt{R_{i}}
$$

It is important to note that by definition, the radius is negative for concave surfaces and positive for convex surfaces [16].

Figure 2 shows the penetration depth, $\delta$, the normal contact force, $F_{\mathrm{N}}$, and the relation forcepenetration for two internally colliding spheres modelled by the Hertz contact law. The generalized stiffness is equal to $6.6 \times 10^{10} \mathrm{~N} / \mathrm{m}^{1.5}$.

It is apparent that the Hertz contact law given by equation (2) cannot be used during both phases of contact (loading and unloading phases), as this model does not take into account the energy dissipation during the process of impact. This is a pure elastic contact model, that is, the contact energy stored during the loading phase is exactly the same restored during the unloading phase. The great advantage of the Hertz law relative to Kelvin-Voigt contact model is its non-linearity. Although the Hertz law is based on the elasticity theory, some studies have been performed to extend the contact law to include energy dissipation. In fact, the most complicated part of modelling impacts is the process of energy transfer. If an elastic body is subjected to a cyclic load, the energy loss due to internal damping causes a hysteresis loop in the force-penetration diagram.

Hunt and Crossley [9] showed that the linear spring-damper model does not represent the physical nature of energy transferred during the impact. Instead, they represent the contact force by the Hertz force-penetration law with a non-linear viscous-elastic element. This approach is valid for direct central and frictionless impacts. On the basis of Hunt and Crossley's work, Lankarani and Nikravesh [6] developed a contact force model with hysteresis damping for impact in multi-body systems. The model uses the general trend of the Hertz contact law, in which a hysteresis damping function is incorporated with the intent to represent the energy dissipated during the impact. Lankarani and Nikravesh [6] suggested separating the normal contact force into elastic and dissipative components as

$$
F_{\mathrm{N}}=K \delta^{n}+D \dot{\delta}
$$

where the first term of the right-hand side is referred to as the elastic force and the second term accounts for the energy dissipated during the impact. In equation (6), the quantity $D$ is a hysteresis coefficient and $\dot{\delta}$ is the relative impact velocity. The hysteresis coefficient is written as a function of penetration as [6]

$$
D=\chi \delta^{n}
$$

in which the hysteresis factor $\chi$ is given by [6]

$$
\chi=\frac{3 K\left(1-c_{\mathrm{e}}^{2}\right)}{4 \dot{\delta}^{(-)}}
$$

where $\dot{\delta}^{(-)}$is the initial impact velocity. The normal contact force is finally expressed as

$$
F_{\mathrm{N}}=K \delta^{n}\left[1+\frac{3\left(1-c_{\mathrm{e}}^{2}\right)}{4} \frac{\dot{\delta}}{\dot{\delta}^{(-)}}\right]
$$

where the generalized parameter $K$ can be evaluated by equations (3) and (4), $c_{\mathrm{e}}$ the restitution coefficient, $\dot{\delta}$ the relative penetration velocity, and $\dot{\delta}^{(-)}$the initial impact velocity. The use of the damping scheme included in this model implies the outcome illustrated in Fig. 3 in which the penetration depth, $\delta$, normal contact force, $F_{\mathrm{N}}$, and hysteresis of an impact between two internal spheres are presented. The generalized stiffness used is $6.6 \times 10^{10} \mathrm{~N} / \mathrm{m}^{1.5}$.

Equation (9) is only valid for impact velocities lower than the propagation velocity of elastic waves

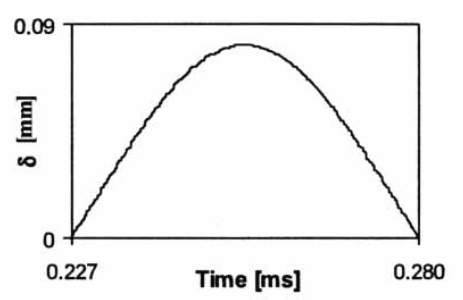

(a)

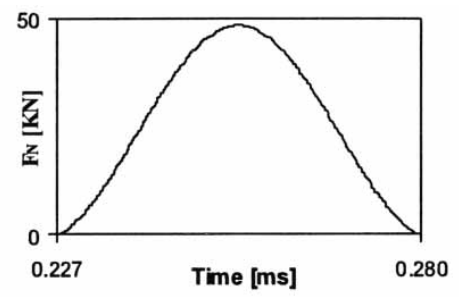

(b)

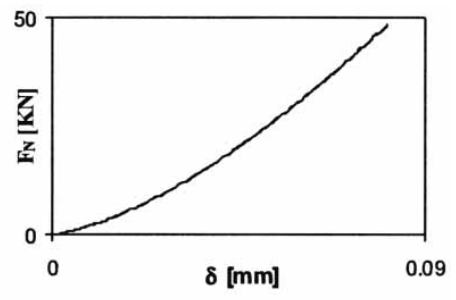

(c)

Fig. 2 Internally colliding spheres modelled by the Hertz contact law: (a) penetration depth, $\delta$; (b) normal contact force, $F_{\mathrm{N}}$; (c) force-penetration ratio 


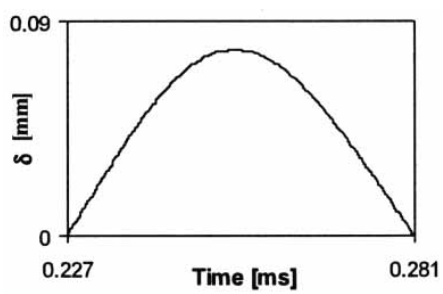

(a)

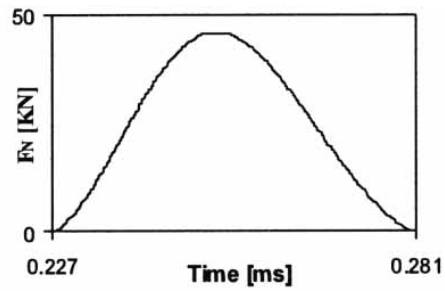

(b)

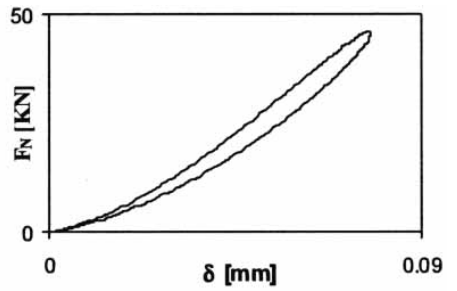

(c)

Fig. 3 Internally colliding spheres modelled by the Lankarani and Nikravesh contact force model: (a) penetration depth, $\delta$; (b) normal contact force, $F_{\mathrm{N}}$; (c) force-penetration relation

across the bodies, i.e. $\dot{\delta}^{(-)} \leqslant 10^{-5} \sqrt{E / \rho}$, where $E$ is the Young modulus and $\rho$ is the material mass density [17]. The quantity $\sqrt{E / \rho}$, velocity of wave propagation, is the larger of propagation velocities of the elastic deformation waves in the colliding bodies. Shivawamy [18] studied theoretically and experimentally the impact between bodies and demonstrated that at low impact velocities, the hysteresis damping is the prime factor for energy dissipation. Impact at higher velocities, exceeding the propagation velocity of the elastic deformation waves, is likely to dissipate energy in a form not predicted by the current model. In a later work, Lankarani and Nikravesh [19] proposed a new approach for contact force analysis, in which the permanent indentation is also included. At fairly moderate or high velocities of collision, especially in the case of metallic solids, permanent indentations are left behind on the colliding surfaces. Hence, local plasticity of the surfaces in contact becomes the dominant source of energy dissipation during impact. Permanent or plastic deformations are beyond the scope of the present work.

\section{NORMAL FORCE MODELS FOR CYLINDRICAL CONTACT SURFACES}

The contact models given by equations (2) and (9) are only valid for colliding bodies with ellipsoidal contact areas. For a cylindrical contact area between two parallel cylinders, a literature search has revealed few and approximate force-penetration relationships. It is worth nothing that line contact assumes a precise parallel alignment of the colliding cylinders. Furthermore, a uniform force distribution over the length of the cylinders is also assumed and boundary effects are neglected. For the case of cylindrical contact forces, some authors suggest the use of the more general and straightforward forcepenetration relation given by equation (9) but with an exponent, $n$, in the range of 1-1.5 [9]. Dietl et al. [20] used the classical solution of contact, presented by Hertz, but with the exponent $n$ equal to 1.08 to model the contact between the journal-bearing elements.

On the basis of the Hertz theory, Dubowsky and Freudenstein [21] presented an expression for the penetration as function of the contact force of an internal pin inside a cylinder as

$$
\delta=F_{\mathrm{N}}\left(\frac{\sigma_{i}+\sigma_{j}}{L}\right)\left[\ln \left(\frac{L^{b}\left(R_{i}-R_{j}\right)}{F_{\mathrm{N}} R_{i} R_{j}\left(\sigma_{i}+\sigma_{j}\right)}\right)+1\right]
$$

where $R_{i, j}$ and $\sigma_{i, j}$ are the parameters shown in equations (3) and (4), $L$ is the length of the cylinder, and the exponent $b$ has a value 3. As equation (10) is a non-linear implicit function for $F_{\mathrm{N}}$, with a known penetration depth, $F_{\mathrm{N}}$ can be evaluated. This is a non-linear problem and requires an iterative scheme, such as the Newton-Raphson method, to solve for the normal contact force, $F_{\mathrm{N}}$.

On the basis of the Dubowsky and Freudenstein contact force model, the solution corresponding to the time variation of the penetration $\delta$, the normal contact force $F_{\mathrm{N}}$, and the force-penetration depth ratio are shown in Fig. 4. In the plots presented, with the case of a pin contact inside a cylinder, the pin and cylinder radii are 9.5 and $10 \mathrm{~mm}$, respectively. The length of the cylinder is equal to $15 \mathrm{~mm}$, and both the pin and cylinder are made of steel.

Goldsmith [15] presented an expression similar to equation (10) but with the value of the exponent $b$ equal to 1 . This value, however, leads to a problem of consistency of the units in the expression. Figure 5 shows the penetration depth $\delta$, the normal contact force $F_{\mathrm{N}}$, and the force-penetration ratio of two internally colliding cylinders modelled with the Goldsmith contact force model. This model shows that the force-penetration ratio is almost linear.

The ESDU-78035 Tribology Series [16] presents some expressions for contact mechanics analysis suitable for engineers' applications. For a circular contact area, the ESDU-78035 model is the same as the pure Hertz law given by equation (2). For rectangular contact, e.g. a pin inside a cylinder, the 


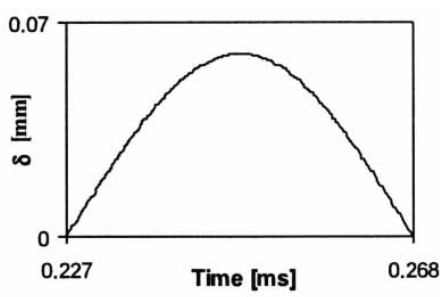

(a)

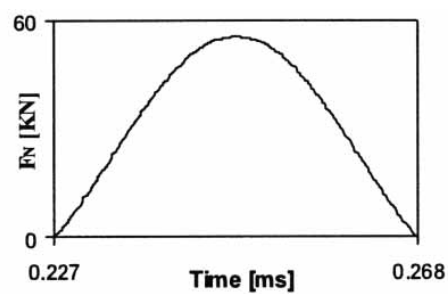

(b)

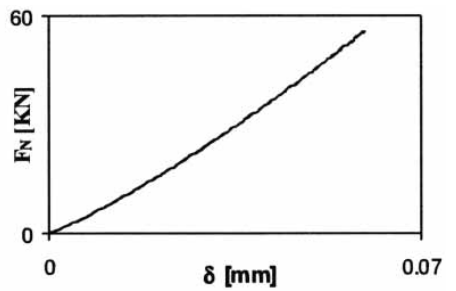

(c)

Fig. 4 Internally colliding cylinders modelled by the Dubowsky and Freudenstein contact force model: (a) penetration depth, $\delta$; (b) normal contact force, $F_{\mathrm{N}}$; (c) force-penetration ratio

expression is given by

$$
\delta=F_{\mathrm{N}}\left(\frac{\sigma_{i}+\sigma_{j}}{L}\right)\left[\ln \left(\frac{4 L\left(R_{i}-R_{j}\right)}{F_{\mathrm{N}}\left(\sigma_{i}+\sigma_{j}\right)}\right)+1\right]
$$

where all quantities have been defined earlier. Figure 6 shows the penetration depth $\delta$, the normal contact force $F_{\mathrm{N}}$, and the force-penetration ratio of two internally colliding cylinders modelled by ESDU-78035, given by equation (11).

A comparison between the spherical and cylindrical contact force models is displayed in Fig. 7. By observing Fig. 7, it can be concluded that the spherical and cylindrical force-penetration relations are reasonably close. Thus, the straightforward force-penetration relation proposed by Lankarani and Nikravesh, given equation (9), is largely used [22-24] for mechanical contacts because of its simplicity and easiness in implementation in a computational program and because this is the only model that accounts for the energy dissipation during the impact.

\section{TANGENTIAL-FRICTION FORCE MODELS}

When contacting, bodies slide or tend to slide relative to each other; there are forces generated which are tangential to the surfaces of contact. These forces are usually referred to as friction forces. Three basic facts have been experimentally established, namely: (a) the friction force acts in a direction opposite to that of the relative motion between the two contacting bodies; (b) the friction force is proportional to the normal load on the contact; and (c) the friction force is independent of a nominal area of contact. These three statements constitute what is known as the laws of sliding friction under dry conditions [25]. Luminaries of science such as Coulomb developed friction studies, but there is still no simple model that can be universally used by designers to calculate the friction force for a given pair of bodies in contact. In recent years, there has been much interest on the subject of friction and many research papers have focused on the subject [26-28].

The presence of friction in the contact surfaces makes the contact problem more complicated, as the friction may lead to different modes, such as sticking or sliding. For instance, when the relative tangential velocity of two impacting bodies approaches zero, stiction occurs. Indeed, as pointed out by Ahmed et al. [29], the friction model must be capable of detecting sliding, sticking, and reverse sliding to avoid energy gains during impact. This work was developed for the treatment of impact problems in jointed open-loop multi-body systems. Lankarani [30] extends Ahmed formulation to the analysis of impact problems with friction in any general multi-body systems including both openand closed-loop systems.

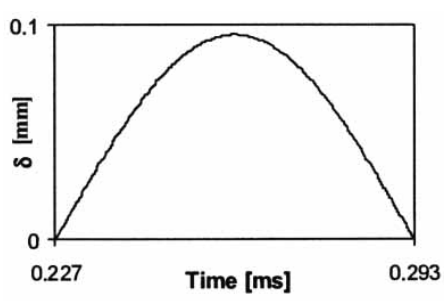

(a)

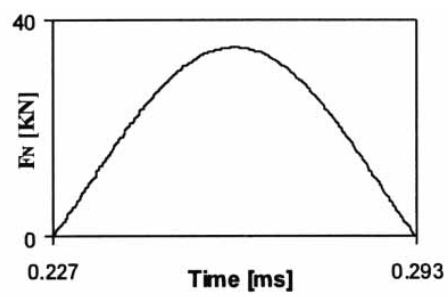

(b)

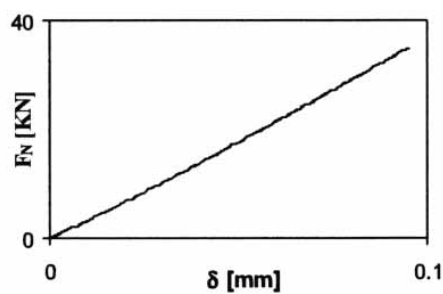

(c)

Fig. 5 Internally colliding cylinders modelled by the Goldsmith contact force model: (a) penetration depth, $\delta$; (b) normal contact force, $F_{\mathrm{N}}$; (c) force-penetration ratio 


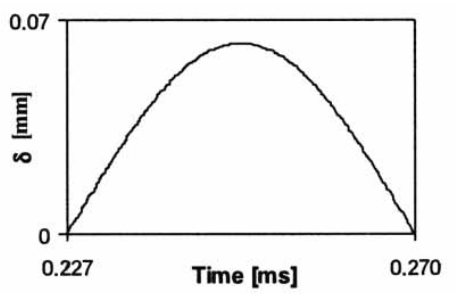

(a)

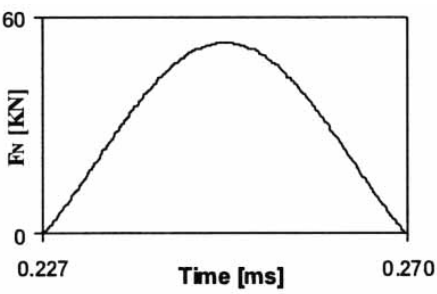

(b)

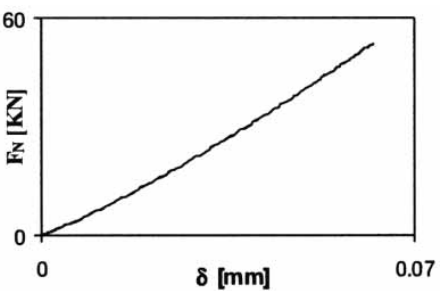

(c)

Fig. 6 Internally colliding cylinders modelled by ESDU-78035 contact force model: (a) penetration depth, $\delta$; (b) normal contact force, $F_{\mathrm{N}}$; (c) force-penetration ratio

The Coulomb friction law of sliding friction can represent the most fundamental and simplest model of friction between dry contacting surfaces. When sliding takes place, the Coulomb law states that the tangential friction force $F_{\mathrm{T}}$ is proportional to the magnitude of the normal contact force, $F_{\mathrm{N}}$, at the contact point by introducing a coefficient of friction $c_{\mathrm{f}}[\mathbf{3 1}]$. The Coulomb friction law is independent of relative tangential velocity. In practice, this is not true, because friction forces can depend on many parameters such as material properties, temperature, surfaces cleanliness, and velocity of sliding. Therefore, a continuous friction force-velocity relationship is desirable. Furthermore, the application of the original Coulomb friction law in a generalpurpose computational program may lead to numerical difficulties because it is a highly non-linear phenomenon that may involve switching between sliding and stiction conditions.

In the last decades, a number of articles have been devoted to the issue of friction [32-35]. Most of them use the Coulomb friction model with some modification in order to avoid the discontinuity at zero relative tangential velocity and to obtain a continuous friction force. Dubowsky [36] assumed the friction

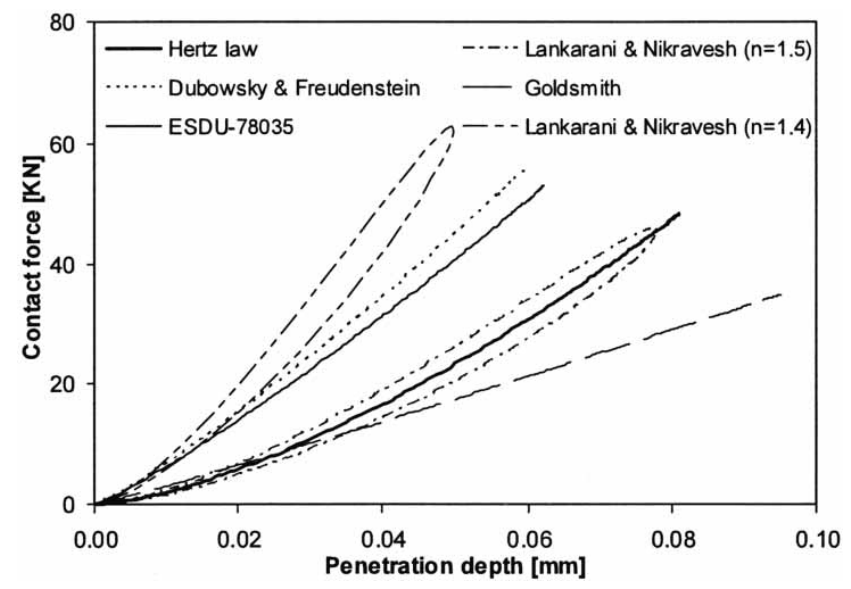

Fig. 7 Force-deformation curves for spherical and cylindrical contact surfaces force to be equal to a constant value opposing the direction of velocity, given by

$$
F_{\mathrm{T}}=-c_{\mathrm{c}} F_{\mathrm{N}} \frac{v_{\mathrm{T}}}{v_{\mathrm{T}}}
$$

where $c_{\mathrm{c}}$ is an empiric friction parameter independent of normal contact force, $F_{\mathrm{N}}$ the normal contact force, $\boldsymbol{v}_{\mathrm{T}}$ the vector of relative tangential velocity, and $v_{\mathrm{T}}$ the magnitude of the relative tangential velocity. This model does not take the effect of zero velocity into account, that is, it has the disadvantage of an infinite gradient at null relative tangential velocity. This causes computational difficulties in the integration process, as the force instantaneously changes from $-F_{\mathrm{T}}$ to $+F_{\mathrm{T}}$. This model is qualitatively illustrated in Fig. 8(a), which shows the Coulomb friction force versus relative tangential velocity.

A friction model with better numerical features is found in Rooney and Deravi [34], which calculated the friction force from two sets of equations. When the relative tangential velocity is not close to zero, the Coulomb friction law is given by

$$
F_{\mathrm{T}}=-c_{\mathrm{f}} F_{\mathrm{N}} \frac{v_{\mathrm{T}}}{v_{\mathrm{T}}}
$$

and when the relative tangential velocity of the contacting bodies is close to zero, the friction force

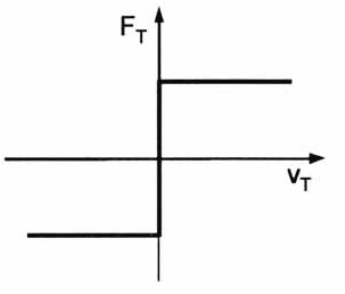

(a)

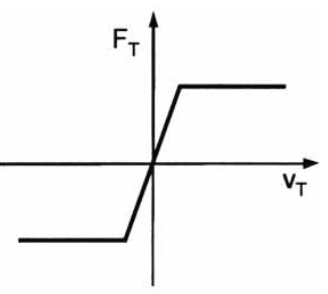

(b)
Fig. 8 (a) standard Coulomb's friction law and (b) Rooney and Deravi friction force 
is a value within a range given by

$$
-c_{\mathrm{f}} F_{\mathrm{N}}<F_{\mathrm{T}}<c_{\mathrm{f}} F_{\mathrm{N}}
$$

where $c_{\mathrm{f}}$ is the coefficient of friction, $v_{\mathrm{T}}$ the relative tangential velocity, and $F_{\mathrm{N}}$ the normal contact force, which is always positive. This model is schematically illustrated in Fig. 8(b).

Wilson and Fawcett [37] used the model expressed by equation (13) in the dynamic study of a slidercrank mechanism with a clearance joint between the slider and the guide. More recently, Ravn [38] also used equation (13) to include the friction effect in revolute joints with clearance.

Threlfall [33] proposed another friction force model, in which the transition between $-F_{\mathrm{T}}$ and $+F_{\mathrm{T}}$ is made using a curve as follows

$$
F_{\mathrm{T}}=c_{\mathrm{f}} F_{\mathrm{N}} \frac{\nu_{\mathrm{T}}}{\nu_{\mathrm{T}}}\left(1-\mathrm{e}^{-\left(3 v_{\mathrm{T}} / \nu_{\mathrm{r}}\right)}\right)
$$

where $c_{\mathrm{f}}$ is the friction coefficient, $F_{\mathrm{N}}$ the normal contact force, and $v_{\mathrm{r}}$ a small characteristic velocity as compared to the maximum relative tangential velocity encountered during the simulation. The value of $v_{\mathrm{r}}$ is a specified parameter, which when very small will result in a slowing down of the integration method as it gets closer to the idealized model of Fig. 8(a). In practice, the regulation factor $1-\left[\mathrm{e}^{-\left(3 v_{\mathrm{T}} / \nu_{\mathrm{r}}\right)}\right.$ smoothes out the friction force discontinuity. The shape of this curve is illustrated in Fig. 9(a).

Ambrósio [39] presented another modification for the Coulomb friction law, in which the dynamic friction force is expressed as

$$
F_{\mathrm{T}}=-c_{\mathrm{f}} c_{\mathrm{d}} F_{\mathrm{N}} \frac{v_{\mathrm{T}}}{v_{\mathrm{T}}}
$$

where $c_{\mathrm{f}}$ is the friction coefficient, $F_{\mathrm{N}}$ the normal contact force, $\nu_{\mathrm{T}}$ the relative tangential velocity, and $c_{\mathrm{d}}$ a dynamic correction coefficient, which is

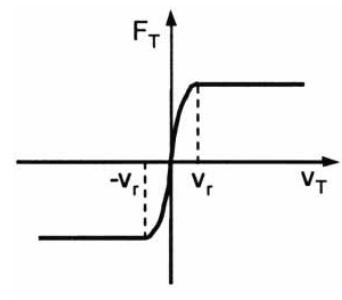

(a)

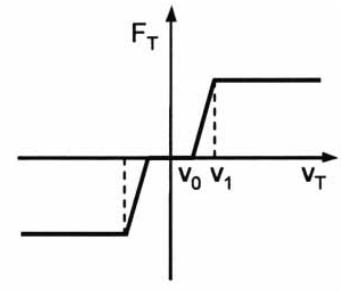

(b)
Fig. 9 (a) Threlfall friction force and (b) Ambrósio friction force expressed as

$$
c_{\mathrm{d}}=\left\{\begin{array}{lll}
0 & \text { if } & v_{\mathrm{T}} \leqslant v_{0} \\
\frac{v_{\mathrm{T}}-v_{0}}{v_{1}-v_{0}} & \text { if } & v_{0} \leqslant v_{\mathrm{T}} \leqslant v_{1} \\
1 & \text { if } & v_{\mathrm{T}} \geqslant v_{1}
\end{array}\right.
$$

in which $v_{0}$ and $v_{1}$ are given tolerances for the velocity. This dynamic correction factor prevents that the friction force changes direction for almost null values of the tangential velocity, which is perceived by the integration algorithm as a dynamic response with high frequency contents, thereby forcing a reduction in the time-step size. The great advantage of this modified Coulomb law is that it allows the numerical stabilization of the integration algorithm. This friction force model, which is illustrated in Fig. 9(b), does not account for other tribological phenomena such as the adherence between the sliding contact surfaces.

The disadvantage when using a friction model such as equation (16), for simulation or control purpose, is the problem of detecting when the relative tangential velocity is zero. A solution for this problem is found in the model proposed by Karnopp, which was developed to overcome the problems with zero velocity detection and to avoid switching between different state equations for sticking and sliding [40]. The drawback with this model is that it is so strongly coupled with the rest of the system. The external force is an input to the model and this force is not always explicitly given. Variations of the Karnopp model are widely used, as they allow efficient simulations, such as the modified Karnopp model by Centea et al. [41] and the reset integrator model by Haessig and Friedland [42].

\section{NUMERICAL ASPECTS IN CONTACT ANALYSIS: COMPUTATIONAL STRATEGY}

In a dynamic simulation, it is very important to find the precise instant of transition between the different sates, that is, contact and non-contact situations. This requires close interaction with the numerical procedure to continuously detect and analyse all situations. If not, errors may built-up and the final results will be inaccurate.

When a system consists of fast and slow components, that is, the eigenvalues are widely spread, the system is designated as being stiff [43]. Stiffness in the system equations of motion arises when the gross motion of the overall multi-body system is combined with the non-linear contact forces that lead to rapid changes in velocity and accelerations. In addition, when the equations of motion are described by a coupled set of differential and 
algebraic equations, the error of the response system is particularly sensitive to constraints violation. Constraints violation inevitably leads to artificial and undesired changes in the energy of the system. However, by applying a stabilization technique, the constraint violation can be reduced and kept under control [44]. During the numerical integration procedure, both the order and the step size are adjusted to keep the error tolerance under control. In particular, the variable step size of the integration scheme is a desirable feature when integrating systems that exhibit different time scales, such as in multi-body systems with impacting bodies [11]. Thus, large steps are taken when the system's motion does not include contact forces, and when impact occurs, the step size is decreased substantially to capture the high frequency response of the system.

One of the most critical aspects in the dynamic simulation of the multi-body systems with collisions is the detection of the precise instant of contact. In addition, the numerical model used to characterize the contact between the bodies requires the knowledge of the pre-impact conditions, that is, the impact velocity and the direction of the plane of collision. The contact duration and the penetration cannot be predicted from the pre-impact conditions because of the influence of the kinematic constraint imposed by all bodies in the overall system motion. Thus, before the first impact, the bodies can freely move relative to each other and, in this phase, the step size may become relatively large. The global motion of the system is then characterized by large translational and rotational displacements. Therefore, if the numerical integration is not handled properly, the first impact between the colliding bodies is often made with a high penetration depth, and, hence, the calculated contact forces become very large.

In order to discuss this issue with some more detail, take, for instance, the relative position between the journal and the bearing in a revolute clearance joint given by [45-47]

$$
\delta=e-c
$$

where $e$ is the absolute eccentricity between the centres of the journal and the bearing and $c$ is the radial clearance. Negative values of $\delta$ mean that there is no contact between the journal and the bearing. Thus, the detection of the instant of contact is when the sign of penetration changes between the two discrete moments in time, $t$ and $t+\Delta t$, that is

$$
\delta(q(t)) \cdot \delta(q(t+\Delta t))<0
$$

Therefore, contact is detected when equation (19) is verified.
An alternative way to determine the instant of contact for multiple time-step size integrators is to use the characteristics of the integration algorithm selected [47]. Supposing that during the normal integration procedure the first contact is detected and that the value of the penetration is higher than a prescribed tolerance. In this case, the integration time step is rejected, not because of error control but because of physical reasons, and a new time step smaller than the previous is tested. The integration process progresses, most probably from a system state for which there is no contact and progresses until contact is detected again. Because the time step is smaller, it is expected that the penetration of this first contact is smaller than the one obtained earlier. The new time steps 'proposed' by the algorithm are rejected until a step is taken, which leads to a penetration within the tolerance. When the 'first' penetration is within the penetration tolerance, it is assumed that such is the moment of the impact and the position, and the relative velocity of the contact points and the direction of the plane of collision are stored. It should be highlighted that with this methodology, the step size can reach smaller values than those required to keep the integration tolerance error under control. Consequently, the numerical system can become unstable if the penetration tolerance imposed is too small. In the present work, the contact detection between the colliding bodies uses this computational strategy [47].

\section{APPLICATION TO THE SIMULATION OF THE SLIDER-CRANK MECHANISM}

The slider-crank mechanism shown in Fig. 10 demonstrates how different impact force models may influence the behaviour of the systems and what the consequences are in terms of the crank reaction moment. There is a revolute clearance joint between the connecting rod and slider, whose value of radial clearance is $0.5 \mathrm{~mm}[\mathbf{4 5}]$. The length and inertia properties of each body are listed in Table 1.

The dynamics of the revolute clearance joint are treated as eccentric impact between the journal

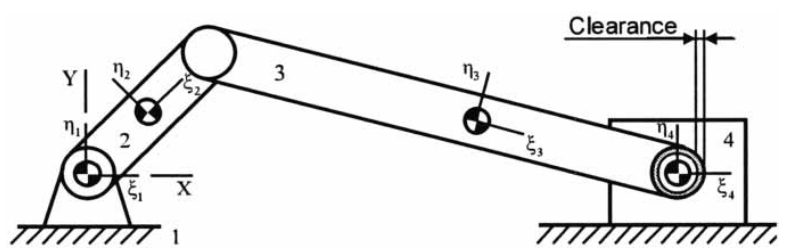

Fig. 10 Slider-crank mechanism with a revolute joint clearance between the connecting rod and slider 
Table 1 Governing properties for the slider-crank mechanism

\begin{tabular}{llll}
\hline $\begin{array}{l}\text { Body } \\
\text { number }\end{array}$ & Length $(\mathrm{m})$ & Mass $(\mathrm{kg})$ & $\begin{array}{l}\text { Moment of } \\
\left.\text { inertia }(\mathrm{kg} \mathrm{m})^{2}\right)\end{array}$ \\
\hline 2 & 0.05 & 0.30 & 0.00001 \\
3 & 0.12 & 0.21 & 0.00025 \\
4 & - & 0.14 & - \\
\hline
\end{tabular}

and the bearing. A mechanism with this type of impact involves both relative normal and relative tangential velocity, when impact between the two bodies is detected, appropriate normal and

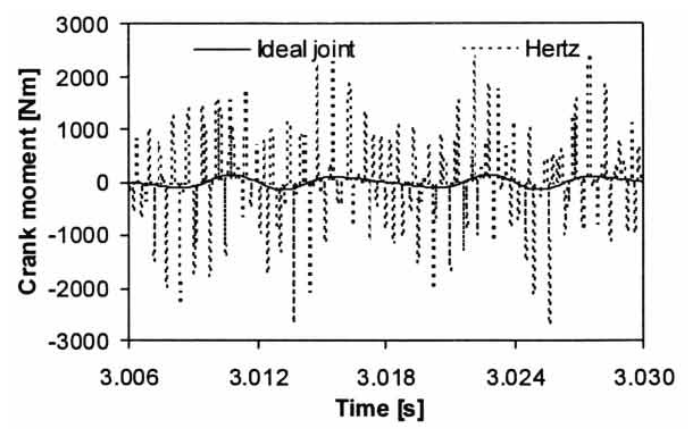

(a)

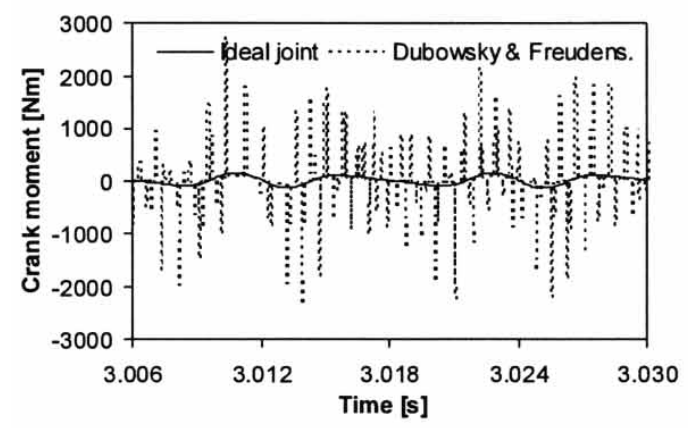

(c)
Table 2 Simulation parameters for the slider-crank mechanism

\begin{tabular}{lc}
\hline Bearing radius & $10.0 \mathrm{~mm}$ \\
Journal radius & $9.5 \mathrm{~mm}$ \\
Journal-bearing length & $15.0 \mathrm{~mm}$ \\
Young's modulus & $207 \mathrm{GPa}$ \\
Poisson's ratio & 0.3 \\
Restitution coefficient & 0.9 \\
\hline
\end{tabular}

tangential contact forces models are applied and the resulting forces are introduced into the system's equations of motion as external generalized forces $[43]$.

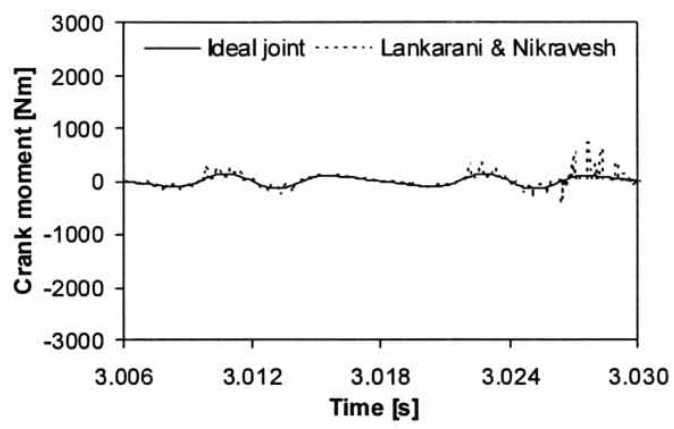

(b)

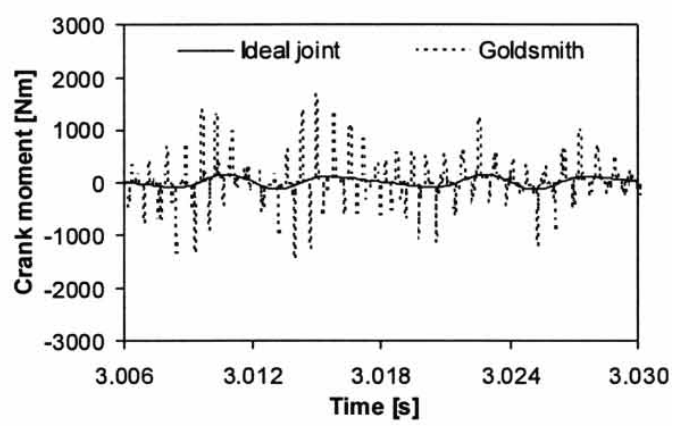

(d)

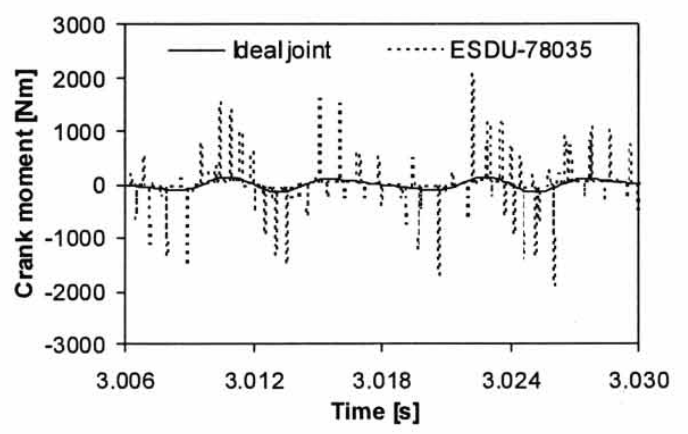

(e)

Fig. 11 Crank moment for the different contact models presented: (a) the Hertz contact law; (b) the Lankarani and Nikravesh model; (c) the Dubowsky and Freudenstein model; (d) the Goldsmith model; (e) ESDU-78035 model 


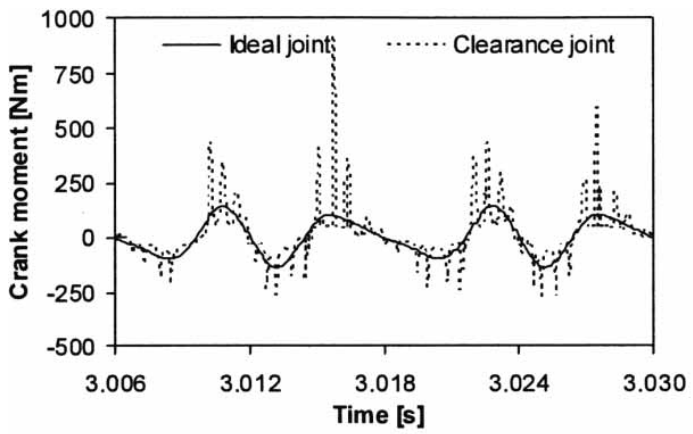

(a)

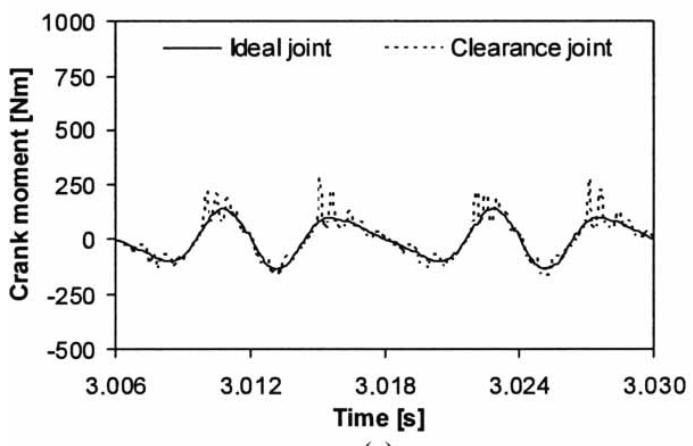

(c)

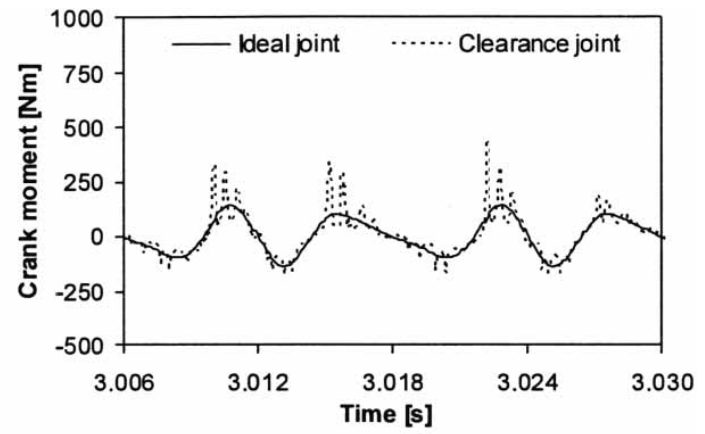

(b)

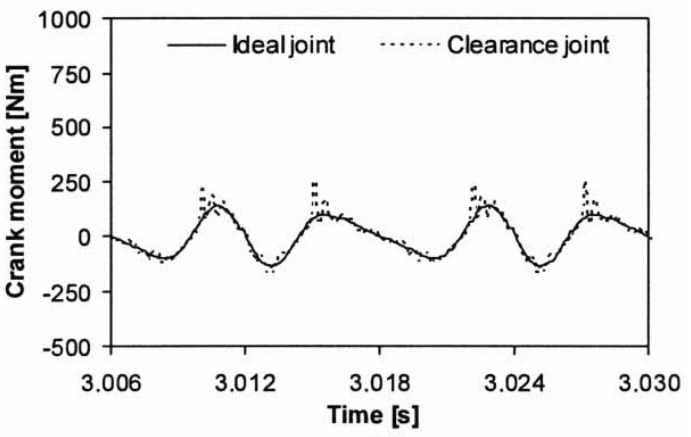

(d)

Fig. 12 Crank moment for Lankarani and Nikravesh contact force with friction effect: (a) $c_{\mathrm{f}}=0.01,(\mathrm{~b}) c_{\mathrm{f}}=0.03,(\mathrm{c}) c_{\mathrm{f}}=0.05$, and (d) $c_{\mathrm{f}}=0.10$

The initial configuration is with the crank horizontal and collinear with the connecting rod. The crank rotates with a constant angular velocity equal to $5000 \mathrm{r} / \mathrm{min}$. Initially, the journal and the bearing centres coincide. Table 2 shows the dynamic parameters used in simulations.

The dynamic simulation of the slider-crank mechanism is performed with the different contact force models presented by equations (2), (9), (10), and (11). In order to keep the analysis simple and to illustrate the influence of the different contact force models in the performance of the slider-crank mechanism, all the bodies are considered to be rigid and the inertia due to the driving motor is neglected. The driving crank moment necessary to maintain a constant crank angular velocity for the different contact models and for the slider-crank with ideal joints are presented in Fig. 11. From Fig. 11, it is clear that for all pure elastic contact models, the level of crank moment is higher when compared with the continuous contact force model proposed by Lankarani and Nikravesh and given by equation (9).

Results for the Lankarani and Nikravesh contact force model, given by equation (9), together with the friction effect are presented. The friction model is given by equation (16). The radial clearance size is equal to $0.5 \mathrm{~mm}$ and four different values for the friction coefficient are used, namely 0.01, 0.03, 0.05, and 0.10 . The friction effect is illustrated by plotting the driving crank moment (Fig. 12). In general, the effect of the friction is to reduce the peaks of the moment values caused by the impact between the colliding bodies.

\section{CONCLUDING REMARKS}

In this work, different contact-impact force models for both spherical and cylindrical shape surfaces collisions in multi-body systems were reviewed. In addition, various types of friction force models based on the Coulomb law were also listed and discussed. Because modelling contact forces plays a crucial role in the analysis of multi-body systems that experience impacts, the contact force model must be computed using suitable constitutive laws that take into account material properties of the colliding bodies, geometric characteristics of the impacting surfaces, and, eventually, the impact velocity. Additionally, the numerical method for the calculation of the contact force should be stable enough to allow for the integration of the mechanical equations of motion. These characteristics are ensured by using a continuous contact force model in which the force and penetration vary in a 
continuous manner and for which some energy dissipation is included. This approach has the further benefit of leading to a behaviour of the variable time-step integrator that is more stable.

In dynamic analysis of multi-body systems, the penetration or deformation is known at every time step from the configuration of the system being the forces evaluated based on the state variables. With the variation of the contact force during the contact period, the dynamic system response is obtained by simply including updated forces into the equations of motion. As the equations of motion are integrated over the period of contact, this approach results in a rather accurate response. This procedure was further improved by including in the time integration scheme a procedure that controls the time step, in order to prevent large penetrations to develop in the initial contact. Furthermore, this methodology accounts for the changes in the system's configuration during the contact period.

Some important conclusions can be drawn from the study presented in this work. Among the spherical shaped contact surfaces, the linear Kelvin-Voigt contact model does not represent the overall nonlinear nature of impact phenomenon. The Hertz relation does not account for the energy dissipation during the impact process. Therefore, the Hertz relation along with the modification to represent the energy dissipation, in the form of internal damping, can be adopted for modelling contact forces in a multi-body system. The cylindrical models are nonlinear and implicit functions, and therefore, they require a numerical iterative procedure to be performed. Furthermore, these models have been proposed as purely elastic not being able to explain the energy dissipation during the impact process. Furthermore, from the comparison between the spherical and cylindrical contact force models, it can be concluded that the spherical and cylindrical force-displacement relations are reasonably close. Therefore, the straightforward force-penetration relation proposed by Lankarani and Nikravesh, with a modification of the pseudo-stiffness parameter in case of cylindrical contact, is largely used for mechanical contacts owing to its simplicity and easiness of implementation in a computational program. Aiding to those advantages, this is the only model that accounts for energy dissipation during the impact process.

\section{ACKNOWLEDGEMENTS}

The research work presented in this article was supported by Fundação para a Ciência e a Tecnologia and partially financed by Fundo Comunitário Europeu FEDER under project POCTI/2001/EME/
38281, entitled 'Dynamic of Mechanical Systems with Joint Clearances and Imperfections.'

\section{REFERENCES}

1 Khulief, Y. A. and Shabana, A. A. Dynamic analysis of constrained system of rigid and flexible bodies with intermittent motion. J. Mech. Trans. Autom. Desi., 1986, 108, 38-45.

2 Rahnejat, H. Multi-body dynamics: historical evolution and application. Proc. Instn Mech. Engrs, Part C: J. Mechanical Engineering Science (special millennium issue), 2000, 214(C1), 149-173.

3 Brach, R. M. Mechanical impact dynamics, rigid body collisions, 1991 (John Wiley and Sons, New York).

4 Maw, N., Barber, J. R., and Fawcett, J. N. The oblique impact of elastic spheres. Wear, 1975, 101-114.

5 Zukas, J. A., Nicholas, T., Greszczuk, L. B., and Curran, D. R. Impact dynamics, 1982 (John Wiley and Sons, New York).

6 Lankarani, H. M. and Nikravesh, P. E. A contact force model with hysteresis damping for impact analysis of multi-body systems. J. Mech. Des., 1990, 112, 369-376.

7 Pfeiffer, F. and Glocker, C. Multi-body dynamics with unilateral constraints, 1996 (John Wiley and Sons, New York).

8 Lankarani, H. M. Canonical equations of motion and estimation of parameters in the analysis of impact problems. PhD Dissertation, University of Arizona, Tucson, Arizona, 1988.

9 Hunt, K. H. and Crossley, F. R. Coefficient of restitution interpreted as damping in vibroimpact. J. Appl. Mech., 1975, 7, 440-445.

10 Ambrósio, J. Rigid and flexible multi-body dynamics tools for the simulation of systems subjected to contact and impact conditions. Eur. J. Solids A/Solids, 2000, 19, S23-S44.

11 Shampine, L. and Gordon, M. Computer solution of ordinary differential equations: the initial value problem, 1975 (Freeman, San Francisco).

12 Zhu, S. H., Zwiebel, S., and Bernhardt, G. A theoretical formula for calculating damping in the impact of two bodies in a multi-body system. Proc. Instn Mech. Engrs, Part C: J. Mechanical Engineering Science, 1999, 213(C3), 211-216.

13 Timoshenko, S. P. and Goodier, J. N. Theory of elasticity, 1970 (McGraw-Hill, New York).

14 Hertz, H. On the contact of solids - on the contact of rigid elastic solids and on hardness (Translated by D. E. Jones and G. A. Schott), Miscellaneous Papers, 1896, pp. 146-183 (Macmillan and Co. Ltd., London, UK).

15 Goldsmith, W. Impact, the theory and physical behaviour of colliding solids, 1960 (Edward Arnold Ltd, London, England).

16 ESDU-78035 Tribology Series. Contact phenomena I: stresses, deflections and contact dimensions for normally- loaded unlubricated elastic components, 1978 (Engineering Sciences Data Unit, London, UK). 
17 Love, A. E. H. A treatise on the mathematical theory of elasticity, 4th edition, 1944 (Dover Publications, New York).

18 Shivawamy, S. Modeling contact forces and energy dissipation during impact in multibody mechanical systems. PhD Dissertation, Wichita State University, Wichita, Kansas, 1997.

19 Lankarani, H. M. and Nikravesh, P. E. Continuous contact force models for impact analysis in multibody systems. Non-linear Dynam., 1994, 5, 193-207.

20 Dietl, P., Wensing, J., and van Nijen, G. C. Rolling bearing damping for dynamic analysis of multi-body systems - experimental and theoretical results. Proc. Instn Mech. Engrs, Part K: J. Multibody Dynamics, 2000, 214(K1), 33-43.

21 Dubowsky, S. and Freudenstein, F. Dynamic analysis of mechanical systems with clearances, Part 1: formulation of dynamic model. J. Eng. Indus., Ser. B, 1971, 93(1), 305-309.

22 Bottasso, C. L., Citelli, P., Taldo, A., and Franchi, C. G. Unilateral contact modeling with adams. In International ADAMS User's Conference, Berlin, Germany, 17-18 November, 1999, p. 11.

23 Pedersen, S., Hansen, J., and Ambrósio, J. A novel roller-chain drive model using multibody dynamics analysis tools. Virtual nonlinear multibody systems, Nato advanced study institute, (Ed. W. Schiehlen and M. Valásek), Prague, Czeck Republic, June 23-July 3 2002, Vol. 2, pp. 180-185.

24 Silva, M. P. T. and Ambrósio, J. A. C. Human motion analysis using multi-body dynamics and optimization tools, Technical Report IDMEC/CPM - 2004/001, Instituto Superior Técnico of the Technical University of Lisbon, Lisbon, Portugal, 2004.

25 Stolarski, T. A. Tribology in machine design, 1990 (Butterworth-Heinemann, Oxford, UK).

26 Keller, J. B. Impact with friction. J. Appl. Mech., 1986, $53,1-4$.

27 Wang, Y. and Manson, M. Two-dimensional rigid-body collisions with friction. J. Appl. Mech., 1992, 59, 635-642.

28 Han, I. and Gilmore, B. J. Multi-body impact motion with friction-analysis, simulation, and validation. J. Mech. Des., 1993, 115, 412-422.

29 Ahmed, S., Lankarani, H. M., and Pereira, M. F. O. S. Frictional impact analysis in open-loop multi-body mechanical system. J. Mech. Des., 1999, 121, 119-127.

30 Lankarani, H. M. A Poisson-based formulation for frictional impact analysis of multi-body mechanical systems with open or closed kinematic chains. J. Mech. Des., 2000, 115, 489-497.

31 Greenwood, D. T. Principles of dynamics, 1965 (Prentice-Hall, Englewood Cliffs, New Jersey).

32 Bagci, C. Dynamic motion analysis of plane mechanisms with coulomb and viscous damping via the joint force analysis. J. Eng. Indus., Ser. B, 1975, 97(2), 551-560.

33 Threlfall, D. C. The inclusion of Coulomb friction in mechanisms programs with particular reference to DRAM. Mech. Mach. Theory, 1978, 13, 475-483.

34 Rooney, G. T. and Deravi, P. Coulomb friction in mechanism sliding joints. Mech. Mach. Theory, 1982, 17, 207-211.
35 Haug, E. J., Wu, S. C., and Yang, S. M. Dynamics of mechanical systems with coulomb friction, stiction, impact and constraint addition-deletion - I theory. Mech. Mach. Theory, 1986, 21(5), 401-406.

36 Dubowsky, S. On predicting the dynamic effects of clearances in planar mechanisms. J. Eng. Indus., Ser. $B, 1974,96(1), 317-323$.

37 Wilson, R. and Fawcett, J. N. Dynamics of slider-crank mechanism with clearance in the sliding bearing. Mech. Mach. Theory, 1974, 9, 61-80.

38 Ravn, P. A Continuous analysis method for planar multibody systems with joint clearance. Multibody Syst. Dyn., 1998, 2, 1-24.

39 Ambrósio, J. A. C. Impact of rigid and flexible multi-body systems: deformation description and contact models. Virtual non-linear multi-body systems, Nato advanced study institute (Eds W. Schiehlen and M. Valásek), Prague, Czech Republic, June 23-July 3 2002, Vol. 2, pp. 15-33.

40 Karnopp, D. Computer simulation of stick-slid friction in mechanical dynamic systems. J. Dyn. Syst., Meas. Contr., 1985, 107, 100-103.

41 Centea, D., Rahnejat, H., and Menday, M. T. Nonlinear multi-body dynamic analysis for the study of clutch torsional vibrations (judder). Appl. Math. Model., 2001, 25, 177-192.

42 Haessig, D. A. and Friedland, B. On the modelling and simulation of friction. J. Dyn. Syst. Meas. Contr., 1991, 113, 354-362.

43 Nikravesh, P. E. Computer-aided analysis of mechanical systems, 1988 (Prentice-Hall, Englewood Cliffs, New Jersey).

44 Baumgarte, J. Stabilization of constraints and integrals of motion in dynamical systems. Comput. Meth. Appl. Mech. Eng., 1972, 1, 1-16.

45 Flores, P., Ambrósio, J., and Claro, J. P. Dynamic analysis for planar multibody mechanical systems with lubricated joints. Multibody Syst. Dyn., 2004, 12, 47-74.

46 Shwab, A. L., Meijaard, J. P., and Meijers, P. A comparison of revolute joint clearance model in the dynamic analysis of rigid and elastic mechanical systems. Mech. Mach. Theory, 2002, 37(9), 895-913.

47 Fernandes, J. P. F. Dynamic analysis of mechanical systems with imperfect kinematic joints. PhD Dissertation, University of Minho, Guimarães, Portugal, 2005.

\section{APPENDIX}

\section{Notation}

$c \quad$ radial clearance $(\mathrm{m})$

$c_{\mathrm{c}} \quad$ empiric friction parameter

$c_{\mathrm{d}} \quad$ dynamic correction coefficient

$c_{\mathrm{e}} \quad$ coefficient of restitution

$c_{\mathrm{f}} \quad$ coefficient of friction

$D \quad$ hysteresis damping parameter $(\mathrm{Ns} / \mathrm{m})$

$E \quad$ Young's modulus of elasticity $\left(\mathrm{N} / \mathrm{m}^{2}\right)$

$e \quad$ eccentricity magnitude (m)

$F_{\mathrm{N}} \quad$ normal contact force $(\mathrm{N})$ 
$F_{\mathrm{T}} \quad$ tangential force $(\mathrm{N})$

$K \quad$ constant of proportionality $\left(\mathrm{N} / \mathrm{m}^{1.5}\right)$

$L \quad$ length (m)

$R \quad$ radius $(\mathrm{m})$

$v_{\mathrm{N}} \quad$ relative normal velocity $(\mathrm{m} / \mathrm{s})$

$v_{\mathrm{r}} \quad$ characteristic velocity $(\mathrm{m} / \mathrm{s})$

$v_{\mathrm{T}} \quad$ relative tangential velocity $(\mathrm{m} / \mathrm{s})$ $\chi \quad$ hysteresis damping factor $\left(\mathrm{Ns} / \mathrm{m}^{2.5}\right)$

$\delta \quad$ penetration depth $(\mathrm{m})$

$\dot{\delta} \quad$ penetration velocity $(\mathrm{m} / \mathrm{s})$

$\dot{\delta}^{(-)} \quad$ initial impact velocity $(\mathrm{m} / \mathrm{s})$

$v \quad$ Poisson's ratio

$\rho \quad$ material mass density $\left(\mathrm{kg} / \mathrm{m}^{3}\right)$

$\sigma \quad$ material parameter $\left(\mathrm{m}^{2} / \mathrm{N}\right)$ 